\title{
Performance Analysis of ZigBee Mesh WSN in Carbon Monoxide Gas Monitoring System
}

\author{
Muhammad Fuad $^{\star 1}$, Muhammad lqbal $^{2}$, Mamat Rahmat ${ }^{3}$, Heru Sukoco ${ }^{4}$, Husin Alatas ${ }^{5}$ \\ ${ }^{1,2,4}$ Department of Computer Science, Faculty of Natural Science and Mathematics, \\ Bogor Agricultural University, Bogor 16680, Indonesia \\ ${ }^{1,2}$ Department of Physics, Faculty of Natural Science and Mathematics, \\ Sriwijaya University, Indralaya 30662, Indonesia \\ ${ }^{5}$ Department of Physics, Faculty of Natural Science and Mathematics, \\ Bogor Agricultural University, Bogor 16680, Indonesia \\ ${ }^{*}$ Corresponding author, e-mail: fuadphysics@gmail.com ${ }^{1}$, hsrkom@ipb.ac.id ${ }^{2}$
}

\begin{abstract}
The need for air pollutant monitoring system is very substantial especially in the developing countries such as Indonesia. In this research, we have performed a test of such system for carbon monoxide gas based on wireless sensor network (WSN) using ZigBee. This system is working with a mesh topology where each sensor node can communicate with one another. There are seven nodes that serve as sensor nodes and one node serving as Coordinator. Each sensor node has five components that represent of gas sensors. We measure three performance metrics during the test, i.e. throughput, delay, and packet loss. The system has been successfully implemented which is capable of displaying information in real time. The experiment resulted in an average carbon monoxide value of $25.1 \mathrm{ppm}$ and showed a good performance. It showed a throughput more than $1.017 \mathrm{kbps}$, delay and packet loss ratio less than $409 \mathrm{~ms}$ and $5 \%$, respectively.
\end{abstract}

Keywords: WSN, air pollutant, CO, performance metrics, ZigBee

Copyright $(2015$ Institute of Advanced Engineering and Science. All rights reserved.

\section{Introduction}

Air pollution is one of the main factors that affecting the quality of human life in the big cities, especially industrial cities, where pollution is one of the causes that affect human health and ecological balances [1-3]. Based on reports from the World Health Organization (WHO), air pollution may cause diseases such as skin and nose irritations. It can also lead to serious problem like heart disorders, lung cancer, pneumonia, bronchitis, and asthma [4]. It is well known that one of the most dangerous gases in air pollution is carbon monoxide (CO) where small amount of this gas can lead to death.

In principle, monitoring of air pollution can be carried out directly by installing gas sensors in various urban areas. The system of wireless sensor network (WSN) is one of the technologies that can be used for monitoring air pollution. WSN is a network technology which integrates sensor nodes to form a wireless network for observations in a region [5, 6]. WSN is one of the rapidly evolving technologies lately [7, 8]. The advantage of this system is its simplicity to be applied in various fields of sciences. For example in the field of physics, WSN can be implemented to observe various physical parameter remotely such as temperature, humidity, vibration, seismic events, gas concentration in the air and so on [5, 9].

One of the main components that can be used in WSN technology is ZigBee which is a communication standard protocol of radio frequency (RF) based on IEEE 802.15.4 [10-12]. This protocol is widely used for automations and wireless networks system applications. ZigBee has several advantages such as low power consumption [13]. In addition, ZigBee can also be considered to minimize cost and low-power connectivity of equipments that require batteries to live for months more years [14], but does not require high speed data transfer such as bluetooth. Therefore, the implementation of ZigBee in a mesh network is more useful than bluetooth.

ZigBee supports three kinds of network topologies namely star, cluster tree and mesh network topology. A star network is a centralized network which forms a direct communication to 
the Coordinator. In cluster tree and mesh network, every ZigBee device can communicate with each other both directly or through its neighbors. This network is formed by one ZigBee Coordinator and multi ZigBee Routers. In the mean time, for a cluster tree network, the Coordinator and Routers serve as beacons. However, in a mesh network, regular beacons are not allows. A mesh network is formed by itself only when needed [15]. Beacons are an important mechanism to support power management. Therefore, the cluster tree network is preferred, especially when energy saving is a desirable feature [16]. But, for delivering continuous data, the mesh network is much better [17]. By testing the application of the mesh topology in a field application, it is expected to obtain information about the advantages and disadvantages of mesh networks which mostly can only be tested through simulation.

This study is aimed to examine the quality service performance of ZigBee on a mesh network topology of a WSN CO gas monitoring system. The results of this study are important because it provides information on how well the corresponding system works.

\section{Research Method}

\subsection{Study Area}

The research was conducted in Bogor Agricultural University campus, situated in the west part of Bogor city. Sensor nodes were installed at points as shown in Figure 1. They mounted on the side of the road where the main campus vehicle traffic levels are high. It is expected that the measurement results can be at a maximum level of air pollution caused mainly by gas from vehicles.

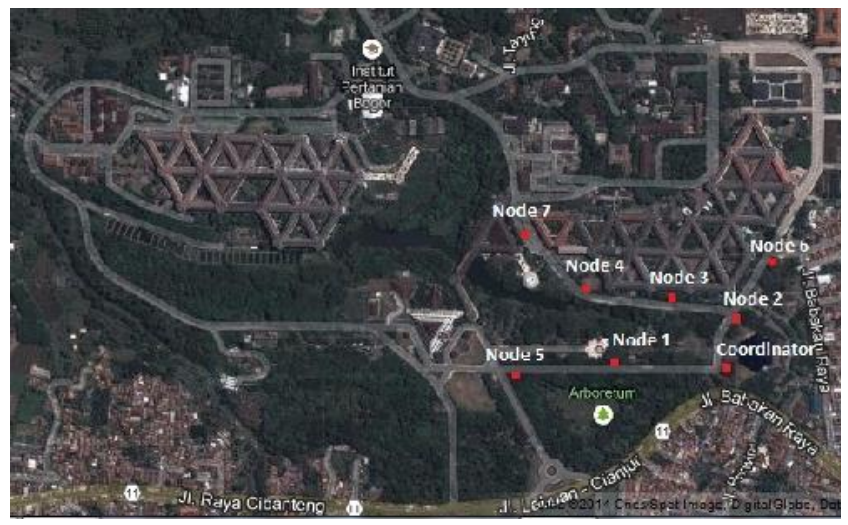

Figure 1. Sensor nodes location

There are eight nodes which can be seen in Figure 1. The nodes consist of 1 Coordinator, 4 Routers and 3 End Devices. The Coordinator serves as a network-forming system that organized communication in the network, and it is also act as a sink. While Routers and End Devices act as a functioning unit that take the measurement data. The distance of nodes is in a range $100 \mathrm{~m}$ to $120 \mathrm{~m}$. This is in accordance with the device specifications. The distance between nodes to the Coordinator can be seen in Table 1.

Table 1. Sensor nodes distanced

\begin{tabular}{ccccccccc}
\hline & Node 1 & Node 2 & Node 3 & Node 4 & Node 5 & Node 6 & Node 7 \\
\hline Function & Router & Router & Router & Router & End Device & End Device & End Device \\
Distance from Coordinator & $120 \mathrm{~m}$ & $100 \mathrm{~m}$ & $120 \mathrm{~m}$ & $210 \mathrm{~m}$ & $230 \mathrm{~m}$ & $220 \mathrm{~m}$ & $320 \mathrm{~m}$ \\
\hline
\end{tabular}

Each nodes consists of a Leonardo Arduino, an XBee Series 2, three dummies sensors of air pollution, a $\mathrm{CO}$ gas sensor (MQ-7 series) and a 9 volt alkaline battery as shown in Figure 2. While the Coordinator consists of a USB adapter, a USB cable and a XBee Series 2 (see Figure 3). Figure 4 shows the connection of Coordinator to computer server. 


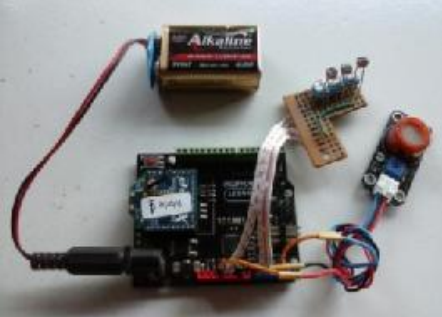

Figure 2. Node sensor unit

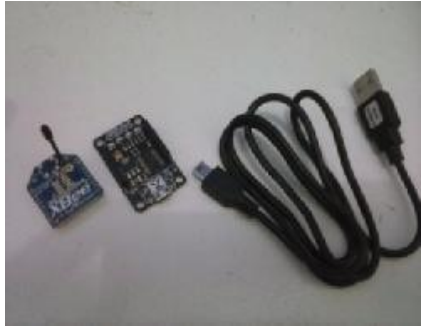

Figure 3. Coordinator unit

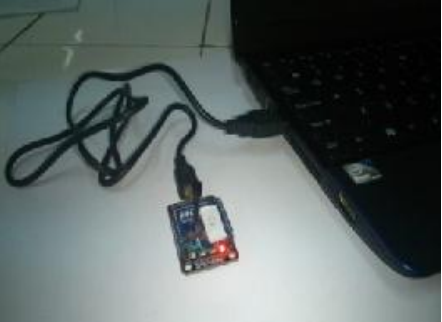

Figure 4. Connection of Coordinator to server

\subsection{System Design}

Mesh topology used in this study can be seen in Figure 5. Three nodes that interconnected directly are nodes 1,3 and 4 . These relationships provide alternative routes for sending data to the Coordinator for its child nodes.

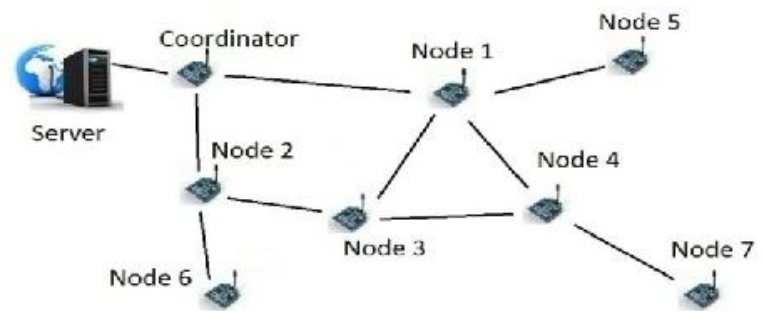

Figure 5. Topology design

\subsection{Data Acquisition}

Data acquisitions were conducted for 4 days with a range time between 09:00am until $17: 00 \mathrm{pm}$. All of ZigBee device were recorded. The numbers of data recording are approximately 300 data. Every ZigBee device will send data to the Coordinator at each second. The measurement data are recorded on a computer server that connected to the Coordinator via the USB cable directly. The data are generated in text files then processed by excel and gnuplot program.

\subsection{Quality of Service (QoS)}

In WSN system the QoS is a set of parameters that indicate the quality of service of a network and the network's abilities to run applications at desired performance. By knowing QoS we can determine the condition of a network and arrange the network which the using application. Some definition of QoS parameters are given in the following.

\subsubsection{Throughput}

This parameter indicates the amount of data packets that received at the destination node than the travel time is written in units of bits per second (bps) [18] as given below:

$$
\text { throughput }=\frac{\text { total recieved bits }}{\text { end time }- \text { start time }}
$$

\subsubsection{Delay}

This parameter represents the time interval between the start of data packets sent to data packets received in the destination node [16] which is defined as follows:

$$
\text { delay }=\frac{\sum(\text { received time }- \text { start time })}{\text { received packets }}
$$




\subsubsection{Packet Loss Ratio}

This parameter calculates the number of missing data on the journey to the destination node [19] which is defined as follows:

$$
\text { packet loss ration }=\frac{\text { sent packet }- \text { arrival packet }}{\text { total packets }} \times 100 \%
$$

\subsection{Air Pollutant Standard Index (ISPU)}

Air pollutant standard index are categorized in five conditions. It can be seen in Table 2 . The ISPU index can be counting by the formula (1).

Table 2. Categories of Air Pollutant Standard Index

\begin{tabular}{ll}
\hline Index & Categories \\
\hline $0-50$ & Fine \\
$50-100$ & Moderate \\
$101-199$ & Bad for healthy \\
$200-299$ & Very bad for healthy \\
$300-$ more & Dangerous \\
\hline
\end{tabular}

\begin{tabular}{|c|c|c|c|c|c|}
\hline ISPU & $\begin{array}{c}24 \mathrm{jam} P M_{10} \\
\mu \mathrm{g} / \mathrm{m}^{3}\end{array}$ & $\begin{array}{c}24 \mathrm{jam} \mathrm{SO}_{2} \\
\mu \mathrm{g} / \mathrm{m}^{3}\end{array}$ & $\begin{array}{c}8 \mathrm{jam} \mathrm{CO} \\
\mathrm{mg} / \mathrm{m}^{3}\end{array}$ & $\begin{array}{c}1 \mathrm{jam} \mathrm{O}_{3} \\
\mu \mathrm{jg} / \mathrm{m}^{3}\end{array}$ & $\begin{array}{c}1 \mathrm{jam} \mathrm{NO_{2 }} \\
\mu \mathrm{g} / \mathrm{m}^{3}\end{array}$ \\
\hline 50 & 50 & 80 & 5 & 120 & \\
\hline 100 & 150 & 800 & 10 & 235 & \\
\hline 200 & 350 & 365 & 17 & 400 & 1130 \\
\hline 300 & 420 & 1600 & 34 & 800 & 2260 \\
\hline 400 & 500 & 2100 & 46 & 1000 & 3000 \\
\hline 500 & 600 & 2620 & 57.5 & 1200 & 3750 \\
\hline
\end{tabular}

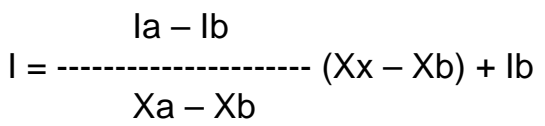

$I=$ Counted ISPU

la $=$ Upper limit ISPU

$\mathrm{lb}=$ Lower limit ISPU

$\mathrm{Xa}=$ Upper limit ambient

$\mathrm{Xb}=$ Lower limit ambient

$\mathrm{Xx}=$ Measurement ambient

\section{Results and Discussion}

From the measured data, we observe the specific behavior exhibits by the Routers and End Devices when sending data to the Coordinator. There are three parameters that indicate the performance of ZigBee mesh networks, namely throughput, delay and packet loss. Each of these parameters will be discussed in this section.

\subsection{Router}

There are 4 nodes working as Routers i.e. node 1, 2, 3 and 4. Each Router will take data and also forward data packets from their child nodes. Router 1 will take data and forward data packets from nodes 5 and 4, while Router 2 will take data and forward data packets from nodes 6 and 3 . In the mean-time, Routers 3 act as a data taker and Router 4 will take data and forward data packet from nodes 7. The networks configuration is shown in Figure 6.

The configuration is determined by observing data packets run to Coordinator while failure treated on Router 1 or Router 2. Failure is conducted by momentarily turn off the Router 1 when the system is running and observe the current of data packets to Coordinator. It is shown that data packets from Router 1 , node 5 , node 4 and node 7 suffer loss. Similar 
treatment was also conducted on Router 2. It is found that data packets from Router 2, nodes 6 and node 3 also suffer loss too.

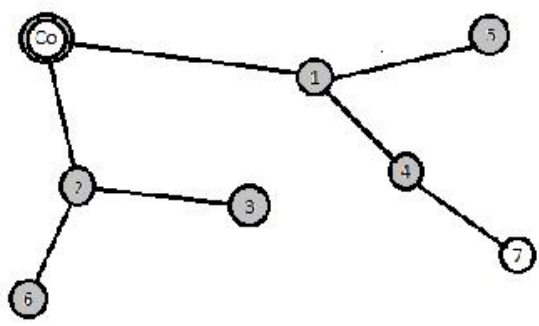

Figure 6. Formed mesh configuration

Throughput on Router 2 has the highest values followed by Router 1,3 and 4 , respectively, as shown in Figure 7 . Routers 1 and 2 have one hop to the coordinator. Since the children of Router 2 is fewer than Router 1 then it is reasonable that its throughput was higher than Router 1. More communication traffic will take more time. Furthermore, throughput values of Router 1 and 2 are higher than Router 3 and 4 because they have two hops to the Coordinator. The average value of throughput can be seen in Table 3.

Table 3. Throughput value on Routers

\begin{tabular}{cccc}
\hline Router & Minimum & $\begin{array}{c}\text { Throughput (kbps) } \\
\text { Maximum }\end{array}$ & Average \\
\hline 1 & 5.859 & 8.157 & 6.794 \\
2 & 6.029 & 11.556 & 9.081 \\
3 & 2.552 & 3.152 & 2.907 \\
4 & 1.558 & 2.337 & 2.110 \\
\hline
\end{tabular}

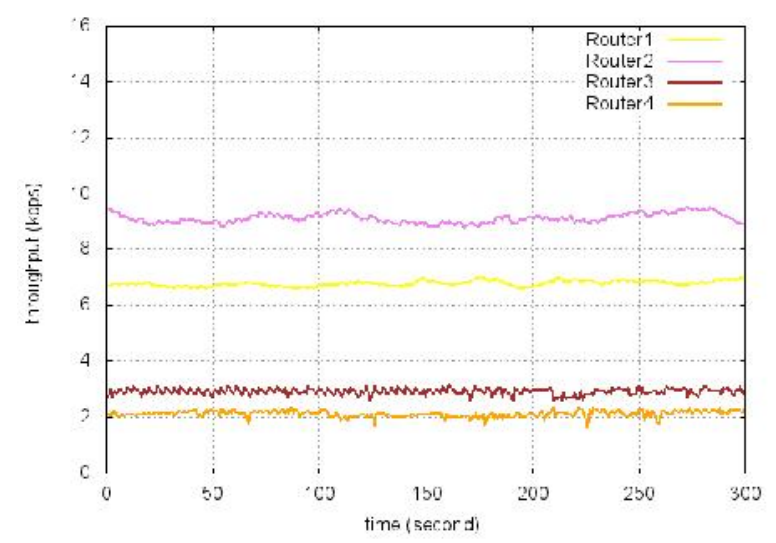

Figure 7. Throughput on Routers

Delay that occurred in the Router can be seen in Figure 8. Router 4 has the highest delay values followed by Router 3,1 and 2, respectively. This delay occurred when data is send to Coordinator in the mean time between sending and receiving data. Routers 3 and 4 have the same number of hop i.e. 2 hops. In the mean time, Router 1 and 2 have also the same number of hop namely 1 hop. Here, because the number of hop determined arrival time to the Coordinator, therefore, Router 4 has delay values more than the others and in addition its through data packet from node 7 . Table 4 shown the average of delay value. 
Table 4. Delay value on Routers

\begin{tabular}{cccc}
\hline Router & Minimum & $\begin{array}{c}\text { Delay }(\mathrm{ms}) \\
\text { Maximum }\end{array}$ & Average \\
\hline 1 & 51 & 71 & 62 \\
2 & 36 & 69 & 47 \\
3 & 132 & 163 & 143 \\
4 & 178 & 262 & 198 \\
\hline
\end{tabular}

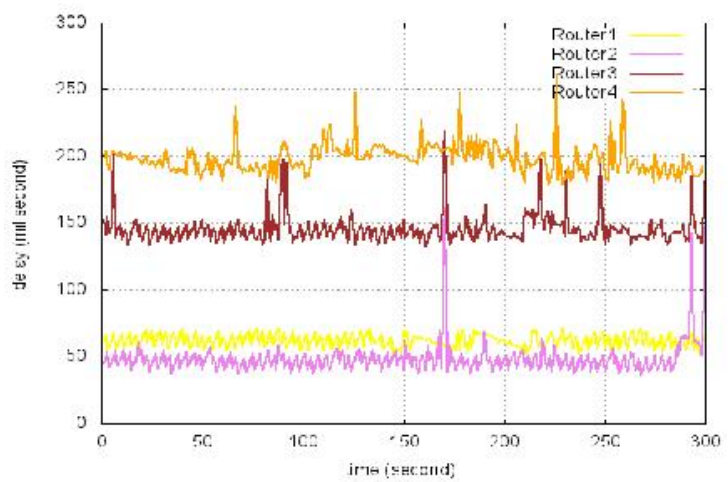

Figure 8. Graph delay on Routers

We observed that there are no missing data packets in Router 1 and 2 during data transmission. On the other hand, there are 5 data packets loss in time of 133, 134, 135, 202 and 275 on Router 3, while for Router 4 there is only one data packet loss in time of 292. The missing packets data occurred due to queue in Coordinator while the new data packet arrived at the queue was already full, the data packet will be wasted, which saw a surge in the delay that occurred at that time. We can count the value of packet loss ratio (PLR) in all Routers by their delay values (see Table 5).

Table 5. Packet Loss Ratio (PLR) on Routers

\begin{tabular}{ccccc}
\hline Router & Sent-packet & Arrival-packet & Total-packet & Packet loss ratio (\%) \\
\hline 1 & 300 & 300 & 300 & 0 \\
2 & 300 & 296 & 300 & 1.33 \\
3 & 300 & 292 & 300 & 2.67 \\
4 & 300 & 287 & 300 & 4.33 \\
\hline
\end{tabular}

\subsection{End Device}

In this section we discuss the throughput, delay and packet loss that occurs at the End Device. There are 3 nodes that working as End Devices namely node 5 as End Device 1, node 6 as End Device 2 and node 7 as End Device 3. The function of End Device are to retrieve data measured by sensors and sending them Coordinator via Routers.

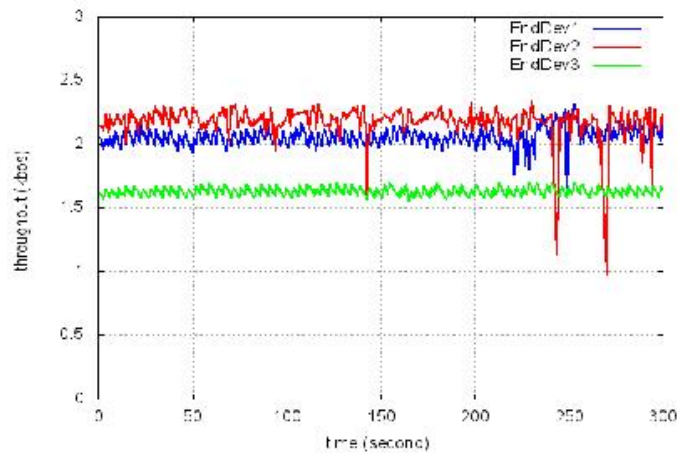

Figure 10. Graph throughput on End Devices 
Figure 10 shows the graph of throughput values on End Devices. Both End Device 1 and 2 have almost same values because of their hop number are the same. End Device 3 has the lowest throughput values becaused of its hop number is more than the others. Table 6 shown the average of throughput value.

Table 6. Throughput value on End Devices

\begin{tabular}{cccc}
\hline End Device & Minimum & $\begin{array}{c}\text { Throughput (kbps) } \\
\text { Maximum }\end{array}$ & Average \\
\hline 1 & 1.651 & 2.405 & 2.136 \\
2 & 1.017 & 2.433 & 2.266 \\
3 & 1.612 & 1.770 & 1.690 \\
\hline
\end{tabular}

Delay on End Devices occurred between 130 to 260 milisecond. Figure 11 depicts the graph of delay value. The highest value occurred at End Devices 3 because of its hop number more than the other ends. Both End Device 1 and End Device 2 have almost the same value. Table 7 shown the average of delay value.

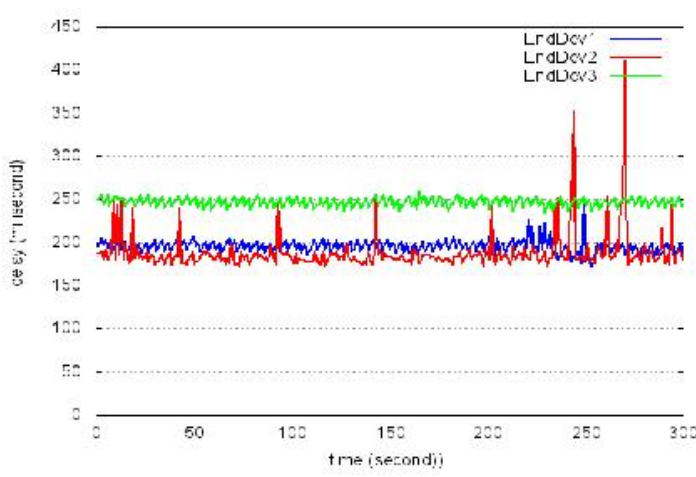

Figure 11. Graph of delay on End Devices

Tabel 7. Delay value on End Devices

\begin{tabular}{cccc}
\hline End Device & Minimum & $\begin{array}{c}\text { Delay (ms) } \\
\text { Maximum }\end{array}$ & Average \\
\hline 1 & 173 & 252 & 195 \\
2 & 171 & 409 & 185 \\
3 & 235 & 258 & 246 \\
\hline
\end{tabular}

The data packets loss only occurred on End Device 2 which 15 data packets. The packet loss ratio can be seen in Table 8 .

Table 8. Packet Loss Ratio (PLR) on End Device

\begin{tabular}{ccccc}
\hline End Device & Sent-packet & Arrival-packet & Total-packet & Packet Loss Ratio (\%) \\
\hline 1 & 300 & 300 & 300 & 0 \\
2 & 300 & 285 & 300 & 5 \\
3 & 300 & 300 & 300 & 0 \\
\hline
\end{tabular}

\subsection{Pollutant Level Measurement Data}

Based on the above performance, we have conducted the measurement of $\mathrm{CO}$ gas concentration using commercial sensor device. The results from the measurement of the corresponding concentration data is given in Table 9. It is demonstrated that the concentration category is fall into "fine" category which means that the environment is not being endangered by the present of $\mathrm{CO}$ gas. 
Table 9. Sample of CO data measurement

\begin{tabular}{ccccc}
\hline \multirow{2}{*}{ Data } & \multicolumn{2}{c}{$\mathrm{CO}$} & \multicolumn{2}{c}{ Pollution Index } \\
& ppm & $\mathrm{mg} / \mathrm{m}^{3}$ & counted & categories \\
\hline 1 & 23 & 0.023 & 0.23 & Fine \\
2 & 23 & 0.023 & 0.23 & Fine \\
3 & 24 & 0.024 & 0.24 & Fine \\
4 & 24 & 0.024 & 0.24 & Fine \\
5 & 25 & 0.025 & 0.25 & Fine \\
6 & 25 & 0.025 & 0.25 & Fine \\
7 & 26 & 0.026 & 0.26 & Fine \\
8 & 27 & 0.027 & 0.27 & Fine \\
9 & 26 & 0.026 & 0.26 & Fine \\
10 & 28 & 0.028 & 0.28 & Fine \\
\hline
\end{tabular}

\section{Conclusion}

We have developed and tested the performance of a $\mathrm{CO}$ gas monitoring system based on wireless monitoring network with mesh topology using ZigBee as its main data transfer protocol. The system has been successfully implemented with capability of displaying information in real time. In addition, the system is able to provide information an average value of $25.1 \mathrm{ppm}$ and have a good performance with more than $1.017 \mathrm{kbps}$ of throughput, no more than 409 ms delay and packet loss ratio less than $5 \%$.

\section{References}

[1] Kasar AR, Khemnar DS, Tembhurnikar NP. WSN based air pollution monitoring system. International Journal of Science and Engineering Application. 2013. 2(4): 55-93.

[2] Mishra SA, Tijare DS, Asutkar GM. Design of energy aware air pollution monitoring system using WSN. International Journal of Advances in Engineering \& Technology. 2011; 1(2): 107-116.

[3] Murthy YN, Sukanya V, Saritha C. Design and development of ZigBee based wireless sensor network for monitoring air pollutants. International Journal of Scientific \& Engineering Research. 2013; 4(3): 1-5.

[4] Lanjewar UM, Shah JJ. Air pollution monitoring \& tracking system using mobile sensors and analysis of data using data mining. International Journal of Advanced Computer Research. 2012; 2(6): 19-23.

[5] G Anastasi, M Conti, M Di Francesco, A Passarella. Energy conservation in wireless sensor networks; A survey. Ad Hoc Networks. 2009; 7: 537-568.

[6] VC Gungor, Lu B, GP Hancke. Opportunities and challenges of wireless sensor networks in smart grid. IEEE Transactions on Industrial Elecronic. 2010; 57(10).

[7] Li F, Wang Y, Li XY, Nusairat A, Wu Y. Gateway placement for throughput optimization in wireless mesh networks. Mobile Netw Appl. 2008; 13: 198-211.

[8] Raju PV, Aravind RS, Kumar BS. Pollution monitoring system using wirelees sensor network in Visakhapatnam. International Journal of Engineering Trends and Technology. 2013; 4(4): 591-595.

[9] IF Akyildiz, W Su, Y Sankarasubramaniam, E Cayirci. Wireless sensors networks; A survey. Computer Networks. 2002; 38(4): 393-432.

[10] Al-Mukhtar M, Hadi TH. Modeling the performance of ZigBee cluster tree wireless sensor networks in presence of failures. Journal of Advanced Computer Science and Technology Research. 2013; 3(3): 116-126.

[11] Digi. ZigBee® Wireless Standard. http://www.digi.com/technology/articles/ Wireless-ZigBee. 2011.

[12] Ergen SC. ZigBee/IEEE 802.15.4 Summary. Berkeley (BK): UC Berkeley. 2004.

[13] Aziz M, Alatas H, Seminar KB. Rancang bangun sistem akuisisi data berbasis wireless sensor network (WSN) dan internet access untuk pengukuran indeks standar pencemar udara (ISPU). Disertasi. Bogor: Institut Pertanian Bogor; 2012.

[14] Ji Z. Design of an integrated controller based on ZigBee wireless sensor network. TELKOMNIKA Indonesian Journal of Electrical Engineering. 2013; 11(8): 4414-4421.

[15] Singh S, Tripathi R. Performance analysis of extended AODV with IEEE802.11e HCCA to support QoS in hybrid network. TELKOMNIKA Indonesian Journal of Electrical Engineering. 2014; 12(9): 6644-6650.

[16] Shiuan PM, Yu CT. Quick convergecast in ZigBee beacons-enabled tree-based wireless sensor network. International Journal of Computer Communication. 2008; 31: 999-1011.

[17] Szigeti T, Hattingh C. End to end QoS network design: Quality of Service in LAN's WAN's and VPN. Indianapolis: Cisco Press. 2004

[18] Misra S, Woungang I, Chandra MS. Guide to wireless ad hoc networks. Toronto: Ryerson University. 2009.

[19] Hanzo L, Tafazolli R. A survey of Qos routing solutions for mobile ad hoc networks. IEEE Communications Surveys \& Tutorials. 2007; 9(2): 50-70. 